\title{
La poésie lyrique a-t-elle une valeur épistémique? Esquisse d'une lecture croisée de Charles Batteux et Jacques Bouveresse
}

\author{
Dimitri Garncarzyk \\ CERC, Sorbonne Nouvelle-Paris $3^{*}$
}

On esquisse ici une lecture croisée de développements du théoricien de la littérature Charles Batteux (1713-1780) et du philosophe analytique français Jacques Bouveresse $(* 1940)$ sur la poésie lyrique pour répondre à une question de philosophie de la littérature : la poésie lyrique a-t-elle une valeur épistémique ? L'introduction justifie le rapprochement entre les deux auteurs (qui peut paraître d'abord incongru) et présente la méthodologie de la reconstruction rationnelle, empruntée à la philosophie analytique. La première partie est consacrée à la place (relative) de la poésie lyrique dans l'essai de J. Bouveresse (2008) et les quelques pistes qu'il formule à l'égard du potentiel épistémique de ce genre littéraire ; la seconde partie montre comment certains développements de Batteux (1753) répondent à ces problèmes. La conclusion résume les bénéfices de cette méthode de lecture croisée, qui aboutit à une réelle plus-value théorique sur la question théorique en jeu, et propose des pistes pour poursuivre cette enquête.

Mots-clés : poésie lyrique, rationalisme, Bouveresse, Batteux, esthétique cognitive.

À première vue, l'abbé Charles Batteux (1713-1780) et le philosophe Jacques Bouveresse $(* 1940)$ ont très peu de choses en commun, à part, peutêtre, d'avoir l'un et l'autre été professeurs de philosophie au Collège de France - Batteux, à partir de 1751 (Goujet 1758 : 325) et jusqu'à 1770, sur la chaire de philosophie grecque et latine, et Jacques Bouveresse, de 1995 à 2010, sur la chaire qu'il intitula «Philosophie du langage et de la connaissance ». Sinon, le catholicisme sincère du premier s'accommoderait mal du scepticisme religieux du second ; la réputation de conservateur de Batteux semble peu compatible avec le clair ancrage à gauche de J. Bouveresse ; les champs couverts par leurs travaux ne se superposent quasiment pas - ne serait-ce pas une idée saugrenue que de proposer une lecture croisée de certains de leurs textes?

\footnotetext{
*garncarzyk.dimitri@orange.fr
} 
Il y a pourtant aussi entre Batteux et J. Bouveresse des affinités intellectuelles. Tous deux associent le souci de la rigueur intellectuelle à une veine polémique certaine, le premier contre " le faux goût des novateurs de notre siècle » (Dupuy 1793 : 97), le second contre la « nouvelle philosophie » française de la fin du $\mathrm{Xx}^{\mathrm{e}}$ siècle. La philosophie autrichienne si chère à $\mathrm{J}$. Bouveresse entretient « une sympathie très prononcée pour l'empirisme britannique » et, corrélativement, « une absence presque totale de respect et de sympathie pour les grandes théories philosophiques et les grandes constructions spéculatives » (Bouveresse 2001 : 140) de la philosophie allemande du XIX ${ }^{e}$ siècle qui trouveront, mutatis mutandis, des échos chez Batteux, lequel présente de nettes convergences avec la pensée esthétique de David Hume. Batteux embrassa avec enthousiasme le sensualisme condillacien, se heurta à l'incompréhension prémonitoire de son traducteur allemand Johann Adolph Schlegel (le père des deux romantiques) ${ }^{1}$. Batteux comme Bouveresse pratiquent, selon le mot de Claudine Tiercelin, « un rationalisme d'Aufklärer » (Tiercelin 2012 : 20-24) plutôt que celui de Lumières radicales à la française. Vu sous cet éclairage, qui est plutôt celui de ses contemporains anglais ou centre-européens, Batteux semble beaucoup moins conservateur : il est, à sa façon un peu intempestive, un représentant d'un certain « rationalisme français » (Tiercelin 2012) au XVIII ${ }^{\mathrm{e}}$ siècle.

Si les affinités entre leurs éthos philosophiques réduisent déjà le caractère saugrenu du rapprochement entre Batteux et J. Bouveresse, il faut encore pour le justifier que ces deux auteurs s'expriment sur un objet commun. Batteux est avant tout un théoricien de la littérature et un philosophe de l'art dont l'opus magnum (le Cours de Belles-lettres ${ }^{2}$ ) traite essentiellement des genres poétiques et rhétoriques ; J. Bouveresse a, de son côté, consacré en 2008 un ouvrage à la philosophie de la littérature, intitulé La Connaissance de l'écrivain, qui pose la question de la valeur épistémique de la littérature en se concentrant quasiexclusivement sur le genre romanesque. L'hypothèse ici est que ces deux travaux de philosophie de la littérature peuvent se compléter mutuellement, soit : d'une part, que le travail de J. Bouveresse donnerait une nouvelle actualité à l'œuvre de Batteux (notamment en proposant un vocabulaire contemporain qui permet, par une traduction conceptuelle, d'intégrer les idées de Batteux dans la conversation philosophique du $\mathrm{XxI}^{\mathrm{e}}$ siècle); d'autre part, que la théorie de Batteux permettrait de traiter certains objets littéraires, comme la poésie lyrique, d'une manière com-

\footnotetext{
${ }^{1}$ Pour une discussion (partielle) de ce problème, $c f$. Genette 2004 : 100.

2 Si l'ouvrage de Batteux le plus souvent cité reste Les Beaux-arts réduits à un même principe (Batteux 1746), dès 1747 la première version du Cours de Belles-lettres, en quatre tomes, est venue développer ce « petit ouvrage » et en « porter [1']application plus loin». Se limiter au premier traité limite considérablement la compréhension de la philosophie de la littérature de Batteux, qui la raffina tout au long de sa carrière dans les remaniements successifs du Cours. Le Cours est ici cité dans l'édition de 1753, non définitive, mais particulièrement complète et soignée.
} 
patible avec la réflexion de $\mathrm{J}$. Bouveresse, et ainsi de remédier à la frustration ressentie par les lecteurs de ce dernier qui auraient aimé qu'il traite la poésie.

Si la lecture croisée qu'on propose ici n'est pas si saugrenue, elle n'en procède pas moins d'une épiphanie philosophique que le lecteur ressentira peut-être aussi en constatant, à la lecture des textes de J. Bouveresse et de Batteux cités plus bas, les affinités qui existent entre leur vocabulaire, leurs préoccupations et leurs réponses. La rencontre d'un tel écho est une des joies de la lecture philosophique, et la comparaison plus systématique esquissée ici une façon de prolonger le plaisir fortuit de l'épiphanie pour en tirer un bénéfice conceptuel.

Le plaisir de développer cette épiphanie doit cependant être complété par une méthode. Celle-ci ne saurait être généalogique, dans la mesure où il n’y a aucun signe que J. Bouveresse ait lu Batteux en particulier, ou se soit en général intéressé aux débats esthétiques de la fin de l'époque moderne. Il s'agit plutôt d'acclimater Batteux au XXI ${ }^{\mathrm{e}}$ siècle en le faisant dialoguer avec J. Bouveresse, c'est-à-dire à pratiquer « la méthode de la reconstruction rationnelle », qui consiste « à essayer, autant que possible, de retraduire les textes du passé dans le langage d'aujourd'hui pour pouvoir évaluer l'intérêt que présentent, de notre point de vue, les solutions qu'ils proposent» (Bouveresse $2006: 20$ ). Son extrême opposé serait une pratique de l'histoire des idées qui enfermerait les penseurs du passé dans leur contexte comme des spécimens dans des bocaux, considérant qu'il existe, entre leurs interrogations et les nôtres, toute la différence du mort au vivant. Au contraire, les tenants « de la reconstruction rationnelle soutiennent qu'il y a à la fois une certaine permanence dans les problèmes et un certain progrès dans les réponses » (Bouveresse 2006 : 20-21) : on voudrait montrer ici que la complémentarité entre les interrogations de J. Bouveresse sur la poésie lyrique et la théorie de Batteux est un cas exemplaire à la fois d'une telle permanence et d'un tel progrès.

L'esquisse présentée ici a donc un triple intérêt. Historique, d'abord, parce même en l'absence de filiation directe entre nos deux auteurs, elle est sans doute une contribution à l'archéologie du rationalisme français en fait de pensée de la littérature. Théorique, ensuite, puisque l'intérêt de cette lecture croisée, au-delà du plaisir de l'épiphanie, consiste à produire une réponse à une question comme celle-ci : la poésie lyrique a-t-elle une valeur épistémique? Méthodologique, enfin et surtout, car l'esquisse ne pourra se faire qu'à gros traits ; mais elle devrait montrer l'intérêt et l'efficacité d'une reconstruction rationnelle qui, en acclimatant Batteux au XxI ${ }^{\mathrm{e}}$ siècle, peut contribuer à une esthétique cognitive d'inspiration analytique. 


\section{Poésie lyrique et connaissance rationnelle : les hésitations de J. Bouveresse}

Le problème qui occupe Jacques Bouveresse dans La Connaissance de l'écrivain est celui de la valeur épistémique de la littérature ; autrement dit, de savoir de quel type exactement de connaissance la littérature (et spécifiquement elle) est le vecteur : " Pourquoi avons-nous besoin de la littérature, en plus de la science et de la philosophie, pour nous aider à résoudre certains de nos problèmes ? Et qu'est-ce qui fait exactement la spécificité de la littérature, considérée comme une voie d'accès, qui ne pourrait être remplacée par aucune autre, à la connaissance et à la vérité ? (Bouveresse 2008 : 29-30).

Des raisons, plus ou moins bonnes, de délaisser la poésie dans l'investigation philosophique

Il est évident dès l'avant-propos (Bouveresse 2008 : 9-10) que « la littérature » est, dans l'ouvrage, synonyme en gros de « le roman », dans la mesure où la première interrogation formulée dans l'ouvrage consiste à demander « si le roman ne serait pas capable [...] d'apport à la philosophie pratique une contribution plus directement philosophique » que celle qui consiste simplement à lui fournir des exemples particulièrement élaborés.

Dans son ouvrage, J. Bouveresse entend cerner en quoi la littérature est l'instrument spécifique d'un type particulier de connaissance (morale) : c'est là une réflexion générale sur la littérature à laquelle l'approche analytique du philosophe confère une originalité certaine ; en revanche, cette réflexion considère un corpus très limité à la fois dans le temps et dans les formes, dont les sources littéraires ne comptent que des romans des $\mathrm{XIX}^{\mathrm{e}}$ et $\mathrm{XX}^{\mathrm{e}}$ siècles (le roman le plus ancien cité étant Vanity Fair de William Makepiece Thackeray, qui date de 1847), et se déploie ainsi dans essai sur la puissance morale du genre romanesque depuis le $\mathrm{XIX}^{\mathrm{e}}$ siècle, ce qui témoigne de nettement moins d'originalité.

Le choix de J. Bouveresse n'est pas rare ; il a notamment déjà été fait par un certain nombre de philosophes qui ont inspiré sa réflexion (Martha Nussbaum dans Love's Knowledge ou Vincent Descombes, notamment ${ }^{3}$ ). Ce choix a cependant la particularité de tenir moins à des raisons méthodologiques strictes (resserrer la discussion autour du roman, ou de certaines formes romanesques en particulier) qu'à des motifs contingents. Dans un entretien accordé à $L$ 'Express à l'occasion de la sortie de son livre, J. Bouveresse reconnaît sans ambiguïté qu'une réflexion exhaustive devrait inclure la poésie, et que s'il ne l'a pourtant pas poussée en ce sens, c'est avant tout pour des raisons pragmatiques :

\footnotetext{
${ }^{3}$ Notamment Nussbaum 1990 ; Descombes 1987.
} 
Pour être complet, il aurait fallu parler aussi des autres genres littéraires, et en particulier de la poésie [...]. Je ne sais pas si j'aurai un jour le courage de m'attaquer au problème sous sa forme la plus générale, mais c'est sûrement ce qu'il faudrait faire, ce qui impliquerait en particulier un examen sérieux de la prétention à une forme spéciale et essentielle de connaissance et de vérité que l'on attribue fréquemment à la poésie. Mais c'est une tâche devant laquelle j'ai toujours reculé avec appréhension, notamment à cause du risque que l'on court presque fatalement de heurter de front des convictions et des sentiments qui sont de nature quasiment religieuse. (Blain 2008)

En d'autres termes, la poésie contribue sans doute, comme le roman (et les autres formes de la littérature - J. Bouveresse ne mentionnant jamais non plus les formes dramatiques), à alimenter notre réflexion et notre connaissance morales. Il est par contre aussi possible qu'on lui attribue parfois une puissance cognitive excessive : J. Bouveresse pense visiblement ici à une forme de révélation dont la poésie serait porteuse, au point d'inspirer à certains des « convictions et des sentiments » quasi-religieux. Il y a dans cette allusion un souvenir à peine voilée de la violente dénonciation, à la première page des Règles de l'art, « des “topos" éculés du culte scolaire du Livre ou des révélations heideggéro-hölderliniennes » (Bourdieu 1992 : 9) qui constitueraient, selon Pierre Bourdieu, les incantations par lesquelles certains « humanistes » contemporains défendraient le « bien symbolique » qu'est la littérature contre l'enquête sociologique et scientifique $^{4}$.

Si les limites génériques et chronologiques de la réflexion de J. Bouveresse sont de fait et non de droit, rien n'interdit de la prolonger sur la poésie ou sur d'autres époques, du moment que l'on prend soin de respecter l'exigence bouveresso-bourdieusienne d'une approche rationaliste de la littérature. C'est exactement ce genre de prolongement que la comparaison (incongrue) avec Batteux entend esquisser.

De la connaissance du poète

Si la littérature doit être porteuse de connaissance, estime J. Bouveresse, ce n'est pas d'une connaissance propositionnelle, mais plutôt d'une connaissance pratique, ou (selon l'idée de H. Putnam) une connaissance « conceptuelle », c'està-dire la connaissance que procure l'immersion dans la mimèsis fictionnelle d'une attitude possible face au monde (Bouveresse 2008 : 59-62). Mais ce rôle épistémique de la littérature est une contribution particulière (dont il est possible de

\footnotetext{
${ }^{4}$ Que La Connaissance de l'écrivain soit, en partie, d'inspiration bourdieusienne est immanquable, dans la mesure où le premier chapitre se fonde sur Bourdieu, Benda et Proust (Bouveresse 2008 : 11). Sur les affinités intellectuelles entre Bourdieu et J. Bouveresse, qui ont été collègues au Collège de France, $c f$. Bouveresse 2003.
} 
rendre compte rationnellement) à la connaissance au sens ordinaire (c'est-à-dire la connaissance rationnelle) ; il ne consiste en aucun cas en une succession d'épiphanies ou d'« intuitions » plus ou moins géniales qui, de manière supra-humaine ou infra-rationnelle, anticiperaient ou concurrenceraient les développements ultérieurs des sciences de la nature ou de l'esprit : ce retour du religieux supposerait une « conception dualiste de la connaissance » (la littérature étant alors constituée en un instrument de connaissance alternatif et infra-rationnel). Cela pose un problème assez immédiat pour le traitement de la poésie, dans la mesure où ce genre de pouvoir de révélation est, typiquement, celui que l'on prête depuis l'Antiquité aux poètes (du vates latin au voyant rimbaldien) ; mais c'est aussi, selon J. Bouveresse, le genre de privilège qu'un écrivain comme Robert Musil (1880-1942) n'est pas du tout prêt à reconnaître à la littérature :

Quand je dis qu'il [i.e. Robert Musil] récuse la conception dualiste de la connaissance, je veux dire qu'il ne croit pas que le poète utilise une autre forme de connaissance que celle de l'homme rationnel. «Il n'est, dit-il, ni le "fou", ni le "voyant", ni l'"enfant", ni une autre malformation quelconque de la raison. Il n'utilise pas non plus une autre espèce quelconque de capacité de connaissance que l'homme rationnel. L'homme qui compte est celui qui dispose de la plus grande connaissance des faits et de la plus grande ratio pour les relier : dans un domaine comme dans l'autre. » Les deux domaines en question sont ceux qu'il appelle le « ratiö́de » et le «non-ratioïde ». Le contraire du poète est, dit-il, «l'homme au point fixe $a$, l'homme rationnel dans le domaine ratioïde ». Mais il rejette totalement l'idée que le domaine non ratioïde échappe en quelque sorte par nature à la connaissance et également celle qui veut qu'il ne puisse être connu et maîtrisé que par des méthodes qui sont constitutivement différentes de celles qui s'appliquent au domaine ratioïde. (Bouveresse 2008 : 60-61)

J. Bouveresse trouve chez Musil la confirmation d'une objection qu'il adressait dans Dire et ne rien dire à Gilles Deleuze lorsque ce dernier suggérait que le poète, l'enfant ou le schizophrène produisent un non-sens signifiant (Bouveresse 1997 : 237). À la conception dualiste de la connaissance, Musil oppose une conception moniste de la rationalité : elle est une et indivise, et le critère de tous les discours. Il existe en revanche différents domaines de l'activité cognitive, qui affirment plutôt leur proximité (ou leur distance) avec la rationalité. Mais c'est là une question de forme discursive (c'est le sens du suffixe -ide) qui ne change rien au fait que, comme pratiques cognitives et discursives, ils restent soumis à la raison. Le « spectre » ratioïde va ainsi des discours de « l'homme rationnel dans le domaine ratioïde » (les discours mathématique ou philosophique, par exemple) à ceux du poète ; mais, qu'ils soient plus ou moins « ratiö̈des ", s'ils doivent contribuer à une connaissance, c'est en définitive à la connaissance rationnelle. En d'autres termes, il est purement gratuit, pour le poète, d'affir- 
mer qu'il détiendrait une connaissance particulière qui se situerait totalement endehors du domaine de la raison ; prétendre cela, c'est tâcher de faire passer la forme pour la substance (ou le non-ratioïde pour le non-rationnel).

Que la poésie (par la posture du voyant, la primauté de la forme ou le trobar clus de l'imagination poétique) s'ancre fermement dans le non-ratioïde ne préjuge en rien de sa valeur épistémique, qui relève du rationnel - mais pose peut-être, par contre, la question de savoir quel bénéfice rationnel peut avoir une pratique littéraire aussi délibérément non-ratioïde. S'il a visiblement quelques doutes sur la question, J. Bouveresse formule cependant une hypothèse :

Si l'on se demande ce qui peut rendre une pensée vivante et agissante, ce qui lui confère un pouvoir sur la volonté, ce qui la met en rapport non pas seulement avec l'esprit mais avec le corps lui-même, c'est évidemment du côté de la forme ou, plus exactement, de l'unité indissociable d'un contenu et d'une forme qu'il faut chercher la réponse. La dépendance du contenu par rapport à la forme atteint évidemment son plus haut degré dans la poésie lyrique. Mais Musil ne conclut pas de cela que la poésie utilise un mode de connaissance fondamentalement différent des autres. Le principe de continuité de la vie psychique signifie que, même à l'extrémité poétique du spectre, l'élément intellectuel et le rapport à la réalité restent encore présents. Et dire que l'élément intellectuel reste présent, même quand l'émotion semble avoir absorbé tout le contenu de la connaissance, ne veut pas dire ici qu'il intervient, de toute façon, nécessairement au niveau de l'élaboration. (Bouveresse 2008 : 70-71)

Première remarque : la dimension formaliste de la poésie (celle de la métrique ou des formes fixes e.g.) serait l'expression la plus claire du pouvoir conatif de la littérature. L'idée est cohérente avec la thèse de J. Bouveresse, que la littérature serait dépositaire d'une connaissance pratique : l'idée que la forme poétique permettrait un retour de l'« esprit » vers le « corps » (soit de l'intelligible vers le sensible) suggère qu'elle a toute sa place dans un processus qui vise, à terme, l'action plutôt que l'intellection. S'esquisse là discrètement l'idée que la forme du poème se traduirait efficacement en formes de vie (Lebensformen) wittgensteiniennes 5 .

Seconde remarque : en précisant que « l'élément intellectuel » n'intervient pas « nécessairement au niveau de l'élaboration », J. Bouveresse prend en compte l'asymétrie qui existe entre la production de l'œuvre poétique et sa réception. Cette asymétrie garantit concurremment, du côté du poète (dans le domaine nonratioïde), la légitimité de son inspiration, d'où qu'elle vienne (appel à la Muse,

\footnotetext{
${ }^{5}$ Quoique J. Bouveresse soit un grand wittgensteinien, ce n'est pas lui qui propose ce rapprochement avec les «formes de vie », mais moi. C'est une idée qu'il faudra creuser, mais qui dépasse de loin le cadre de notre esquisse (comme il dépassait celui de La Connaissance de l'écrivain). Sur les formes de vie, $c f$. Wittgenstein $2004: 35$; pour une analyse critique de ce concept, $c f$. Laugier 2015.
} 
contemplation introspective, promenade en forêt, stupéfiants...) ; et du côté de l'herméneute (dans le domaine ratioïde), la légitimité d'une exigence de sens. Si on ne peut pas demander à un poète de se comporter en mathématicien ou en philosophe, on ne peut pas non plus, au nom de l'épiphanie poétique, soustraire par principe la poésie à l'examen rationnel.

\section{L'ode et la raison, ou Charles Batteux et le spectre ratioïde}

Charles Batteux affrontait au XVIII ${ }^{\mathrm{e}}$ siècle, mutatis mutandis, des problèmes très similaires. Un mot sur le contexte dans lequel il a travaillé s'impose avant de se lancer dans la reconstruction rationnelle proprement dite.

\section{Le projet théorique de Batteux : par-delà Anciens et Modernes}

On peut décrire l'œuvre théorique de Batteux comme une longue et minutieuse tentative de proposer à la Querelle des Anciens et des Modernes (telle, au moins, qu'elle s'est remanifestée dans la querelle d'Homère dans les années 1710-17206) une solution médiane, à la fois conservatrice et rationaliste.

Le projet théorique de Batteux est conservateur parce qu'il continue d'accorder aux textes antiques une place prépondérante tant dans la culture poétique que dans le socle théorique du lettré. Dès son tout premier traité en 1746, Batteux entendait établir « le principe de l'imitation que le Philosophe grec établit pour les beaux-arts » (Batteux 1753i : vii), soit la mimèsis aristotélicienne ; et il sera le seul traducteur français de la Poétique au XVIII siècle (Batteux 1771). Mais ce projet est aussi rationaliste parce que Batteux entend aussi satisfaire à l'une des exigences les plus importantes des Modernes, qui est précisément celle d'introduire de la rationalité dans les lettres (ce qui l'amènera à critiquer comme irrationalistes certaines attitudes des Anciens ${ }^{7}$ ). Là où les Modernes du XVII et du début du XVIII ${ }^{e}$ siècle étaient cartésiens, Batteux adoptera les derniers développements de la philosophie de l'esprit en s'appropriant le sensualisme (il prononcera d'ailleurs en 1768 le discours de réception de Condillac à l'Académie française).

La querelle n'a pas été seulement un débat patrimonial sur la valeur des poèmes antiques, mais aussi un débat théorique fondamental sur la valeur de la poésie en soi et, notamment, de sa valeur épistémique. Quand un critique reproche à Homère ses invraisemblances au nom de l'esprit de géométrie, la philologue Anne Dacier s'écrie en 1714 : « quel fléau, pour la poésie, qu'un géomètre ! ; ce à quoi l'abbé Terrasson, un critique Moderne, répliquera : "la géométrie semble

\footnotetext{
${ }^{6}$ Sur la querelle d'Homère en général, $c f$. Norman 2011, ou pour un aperçu, Chevrel 2014 : 276-291.

${ }^{7}$ Pour des exemples concrets, $c f$. Garncarzyk 2017.
} 
avoir porté malheur à ce poète de tous temps, et les deux plus redoutables censeurs qu'il ait eus dans l'Antiquité, savoir Pythagore et Platon, étaient géomètres. C'est en ce sens qu'on doit s'écrier : "quel fléau, non pour la poésie, mais pour Homère, qu'un géomètre !" » (cités par Norman 2011 : 156 -157).

L'abbé Terrasson distingue Homère en particulier et la poésie en général pour répliquer à Anne Dacier que la poésie ne présuppose pas nécessairement l'irrationalisme, suggérant que la pratique moderne saurait concilier poésie et esprit de géométrie. C'est une concession que tous les Modernes ne sont pas prêts à faire, en particulier concernant la poésie lyrique. Antoine Houdar de La Motte (dont l'adaptation de l'Iliade en 1714 avait commencé la Querelle d'Homère), parlant de sa propre ode « L'ombre d'Homère », écrivait ainsi en 1715 :

[...] j'ai souvent ri tout seul de cet orgueil lyrique dans le temps même que je m'y prêtais, et j'en demande encore pardon aux gens raisonnables.

Et d'ailleurs, de quoi un poète s'enorgueillirait-il ? D'un art plus pénible qu'important ; d'exprimer quelquefois avec grâce des choses communes que d'autres pensent et sentent sans en être vains; de quelque facilité à peindre des images, et à rendre des sentiments ? Tout cela bien apprécié, n'est qu'une imagination heureuse, mais qui pour l'ordinaire nuit au jugement, à mesure qu'elle est forte et dominante. (Houdar de La Motte $1715: 21$ )

L'apport discursif de la poésie lyrique au discours psychologique serait ainsi purement ornemental, et son apport épistémique, en vertu d'une sorte de principe d'idempotence, au mieux nul - et même, la plupart du temps, négatif (l'« imagination » ayant tendance à obscurcir le " jugement »). Autrement dit, en écrivant que l'« orgueil » du poète doit faire rire les « gens raisonnables », La Motte identifie (et récuse largement) l'ancrage de la lyrique dans le domaine non-ratioïde.

Chaleur et lumière : le spectre ratioïde selon Batteux

Héritier de ces débats, Batteux s'est efforcé de formuler une théorie aussi rationnelle que possible de la poésie lyrique. On pourrait, de prime abord, considérer le développement qui suit comme une variation philosophisante, mais topique au fond, sur les vers bien connus de l'Ars poetica d'Horace : "Omne tulit punctum, qui miscuit utile dulci,/Lectorem delectando, pariterque monendo - Le point de la perfection est de savoir mêler l'utile à l'agréable, de savoir plaire et instruire » (Batteux 1771:50-51). Mais on peut aussi le lire comme une reconstruction rationnelle d'Horace au XVIII ${ }^{\mathrm{e}}$ siècle — et, ce faisant, apercevoir la parenté entre la façon dont Batteux se pose le problème du statut épistémique de la lyrique et celle de Bouveresse. 
Les hommes ont une intelligence et une volonté, deux facultés dont les opérations sont des connaissances et des mouvements. Ces opérations ne se séparent guère plus les unes des autres, que les facultés mêmes qui les produisent ne se séparent dans notre âme. Quand nous pensons, nos goûts se mêlent à notre pensée. Quand nous sentons, nos pensées se mêlent dans nos goûts. Ainsi, soit que nous parlions, ou que nous écrivions, il y a ordinairement dans ce que nous disons, de la lumière et de la chaleur : de la lumière, elle tient à l'intelligence et à la pensée ; de la chaleur, elle tient à la volonté, au sentiment, au goût.

J'ai dit « ordinairement », parce qu'il y a des genres où la lumière est seule : par exemple, la géométrie ; et qu'il y en a aussi d'autres où la chaleur est seule aussi, comme la musique. Mais ici nous ne parlons que des ouvrages en vers ou en prose, qui ont pour objet de plaire ou d'instruire en même temps : ce qu'on appelle des ouvrages de goût. Il y a nécessairement dans ces sortes d'ouvrage lumière et chaleur : parce que sans l'une le lecteur pourrait s'égarer : et que sans l'autre il s'ennuierait. (Batteux 1753iv : 6-7)

Ce qui, chez Horace, était un principe d'évaluation (le bon poète sait en même temps instruire et plaire) devient chez Batteux un principe métaphysique qui définit la catégorie des « ouvrages de goût » (ce que nous appelons littérature) : c'est une propriété essentielle de l'œuvre littéraire que de s'adresser à la fois à la faculté intellective de l'âme et à sa faculté active. À la fois, mais aussi plus ou moins : puisque la littérature est par définition un mélange de « chaleur » et de « lumière » (de sensible et d'intelligible), toute œuvre comporte normalement l'une de ces variables en proportion inverse de l'autre. Au-delà des bornes de l'ordre du discours se trouvent la géométrie (purement intelligible et exprimable par les nombres) et la musique (purement sensible et exprimée par les sons). L'existence des grands genres au sein de la littérature est liée à cette vision scalaire : « l'histoire, les dissertations, les argumentations demandent surtout à être claires et lumineuses »; « l'oraison [i.e. les genres oratoires], l'épopée, les drames » combinent les deux qualités de manière variable. Enfin, dans la lyrique, « ce sera toujours à la chaleur à dominer » (Batteux 1753iii : 8). 


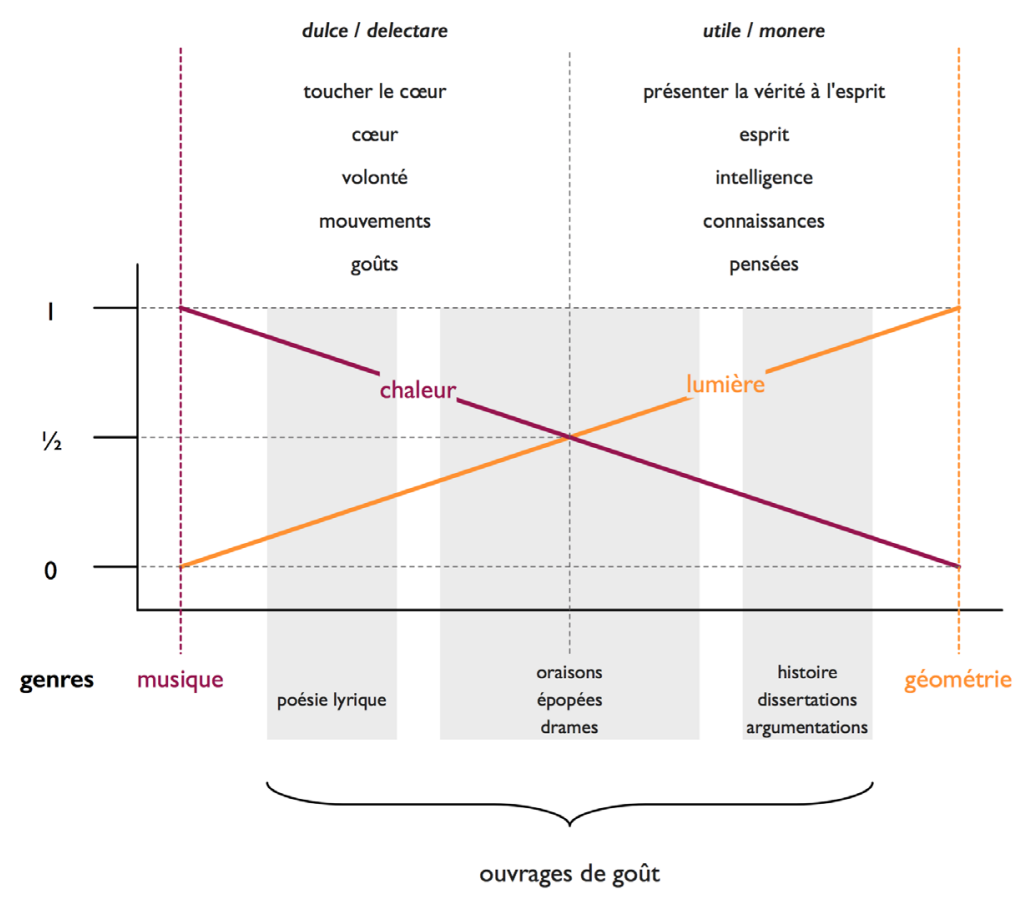

« Principe de continuité de la vie psychique » et genres littéraires (d'après Batteux 1753iii:6-8)

En décrivant l'intrication variable des deux facultés de l'âme, Batteux propose quelque chose de très semblable au «principe de continuité de la vie psychique » selon J. Bouveresse - un « spectre » qui a bien une extrémité rationnelle et une « extrémité poétique »; et s'il ne parle pas du roman (objet exclusif de J. Bouveresse), il fait peu de doute que ce genre se retrouverait auprès de sa cousine l'épopée dans le bloc des genres moyens. Pour simple (et relativement intuitive) qu'elle soit, la théorie de Batteux lue au prisme des remarques de J. Bouveresse est une théorie épistémique des genres littéraires.

\section{Ce que Batteux fait à Bouveresse, et réciproquement}

Si l'on a bien ici l'esquisse d'une méthode, la place manque pour dessiner finement les implications théoriques de cette lecture croisée. Voici donc quelques conclusions (très) provisoires, assorties des grands traits que devra suivre, dans des études ou un livre à venir, ce programme de reconstruction rationnelle. 
Ce que Batteux fait à J. Bouveresse

La poésie lyrique a, dans la réflexion de J. Bouveresse, un statut un peu parasitaire, comme en témoigne à un moment cette parembole : « la littérature - en tout cas le roman, car il serait sûrement difficile de dire la même chose de la poésie lyrique par exemple - peut être une façon de faire de la philosophie » (Bouveresse 2008 : 134). Mais à lire J. Bouveresse à la lumière de Batteux, la poésie lyrique trouve sa place dans une conception cognitive des genres littéraires. L'intuition bouveressienne qu'une « pensée vivante et agissante » qui s'adresserait à la « volonté » est d'autant mieux incarnée dans les textes où forme et contenu sont le plus indissociables, trouve sa contrepartie dans la définition par Batteux de la lyrique comme genre par excellence de la « chaleur» qui échauffe l'âme et la prépare au « mouvement », c'est-à-dire à l'action. La lyrique est ainsi le genre le plus conatif dans l'ordre du discours, celui qui s'adresse (assez remarquablement, plus que l'oraison religieuse ou politique) à la " volonté ».

Simultanément, Batteux rend compte du fait que la lyrique est le genre non-ratioïde par excellence et se caractérise par « les écarts, les digressions, le désordre » et la déliaison logique (on y trouve des " vides entre deux idées »Batteux 1753iii : 18-20). La lyrique joue avec l'extrémité musicale du spectre : comme l'étymologie l'indique, « la poésie lyrique et la musique doivent avoir entre elles un rapport intime » et « ont l'une et l'autre les mêmes objets à exprimer », à savoir « les sentiments du cœur ». Mais la lyrique n'est pas encore pour autant la musique : si la seconde emploie des « sons inarticulés », la première use des « sons articulés ou, ce qui est la même chose, [...] les mots » (Batteux 1753iii : 6). Le fait qu'elle reste, malgré tout, dans l'ordre du langage (« les ouvrages de goût ») implique qu'elle reste soumise au règne de l'intelligible — où l'on retrouve l'objection bouveressienne à l'idée qu'il y aurait des non-sens signifiants. En croisant les vocabulaires et les concepts, on peut ainsi proposer un embryon de réponse à la question de la valeur épistémique de la lyrique, ce qui représente une nette plus-value intellectuelle par rapport à aux lectures historicisantes.

\section{Ce que J. Bouveresse fait à Batteux}

La reconstruction rationnelle est ainsi un travail de traduction d'un langage philosophique passé (qui peut sembler dépassé) dans un langage philosophique contemporain. Que la réflexion d'un philosophe analytique contemporain rencontre certains des mêmes termes qu'employait un esthéticien du XVIII ${ }^{\mathrm{e}}$ siècle témoigne à la fois d'une certaine " permanence dans les problèmes » et d'une réceptivité possible de notre $\mathrm{XXI}^{\mathrm{e}}$ siècle au genre de réponses que pouvait y proposer Batteux. 
L'esthétique contemporaine et son histoire sont largement tributaires du « Grand Récit» de la naissance de l'esthétique au XVIII' siècle, qui culminerait en 1790 avec la troisième Critique de Kant (Pouivet 2006) — autrement dit, « le passage d'une métaphysique du beau à une analytique du goût » (Kremer 2008 : 54). Batteux serait ainsi un conservateur génial qui, en proposant sa définition du goût comme « une connaissance des règles par le sentiment 》 (Batteux 1747 : 102), essaierait tant bien que mal de suivre le cours de l'histoire — soit en se livrant à une Aufhebung hégélienne, qui consiste à « récupérer en le modifiant ce que l'on vient de rejeter » (Rabau 2012 : 145), soit en proposant une conception dualiste de la connaissance (cette définition impliquerait par exemple qu' ' il est vraisemblablement une connaissance légitime qui n'est pas d'ordre cognitif » - Kremer 2008 : 53-54). Le travail de J. Bouveresse fournit les éléments d'un vocabulaire qui fait résonner Batteux avec des interrogations contemporaines et de sortir le spécimen historique de son bocal contextuel (et souvent téléologique) pour jeter en sa compagnie un regard neuf sur nos problèmes esthétiques.

En lisant Batteux de cette façon - comme Roger Pouivet (2006 : 149162) a lu Thomas Reid, par exemple - on donne de nouveaux instruments conceptuels à une esthétique non kantienne, mais cognitive et rationaliste. Cette esthétique serait « une province de l'épistémologie » (Pouivet $2006: 34)$ : la mimèsis, à laquelle Batteux est si attaché, n'est-elle pas aussi un principe cognitif ( « la connaissance n'est jamais une simple reproduction passive de la réalité et implique toujours une créativité d'une certaine sorte »- Bouveresse $2014: 2$ ) ? Ce « goût» si étonnant ne serait-il pas une « vertu esthétique » (Pouivet 2006 : 179-222) ? Autant de questions pour de prochaines lectures !

\section{Références bibliographiques}

Batteux 1747 [1746] : Ch. Batteux, Les Beaux-arts réduits à un même principe, nouvelle édition, Paris : Durand.

Batteux 1753i-iv : Ch. Batteux, Cours de Belles-lettres, ou principe de la littérature, nouvelle édition, Paris : Desaint et Saillant (4 t.).

Batteux 1771 : Ch. Batteux, Les Quatre poétiques d'Aristote, d'Horace, de Vida et de Despréaux, Paris : Desaint et Saillant.

Blain 2008 : J. Blain, « Jacques Bouveresse — "J'ai toujours été profondément révulsé par le moralisme" », L'Express. 〈https://www.lexpress.fr/culture/livre/jacquesbouveresse-j-ai-toujours-ete-profondement-revulse-par-le-moralisme_814050. html.

Bourdieu, 1992 : P. Bourdieu, Les Règles de l'art. Genèse et structure du champ littéraire, Paris : Seuil.

Bouveresse, 1997 : J. Bouveresse, Dire et ne rien dire. L'illogisme, l'impossibilité et le non-sens, Nîmes : Jacqueline Chambon. 
Bouveresse 2001 : J. Bouveresse, Essais II. L'époque, la mode, la morale, la satire, Marseille : Agone. 〈https://www.cairn.info/l-epoque-la-mode-la-morale-la-satire-9782910846466-page-185.htm〉.

Bouveresse 2003 : J. Bouveresse, Bourdieu, savant et politique, Marseille : Agone.

Bouveresse 2006 : J. Bouveresse, Essais V. Descartes, Leibniz, Kant, Marseille : Agone.

Bouveresse 2008 : J. Bouveresse, La connaissance de l'écrivain. Sur la littérature, la vérité et la vie, Marseille: Agone.

Bouveresse 2011 : J. Bouveresse, Essais VI. Les lumières des positivistes, Marseille : Agone.

Bouveresse 2014 : J. Bouveresse, «Y a-t-il une épistémologie de la connaissance littéraire — et peut-il y en avoir une ?». 〈https://hal-univ-diderot.archives-ouvertes.fr/ hal-01184504>, 17/05/2019.

Bouveresse 2016 : J. Bouveresse, Nietzsche contre Foucault. Sur la vérité, la connaissance et le pouvoir, Marseille : Agone.

Chevrel 2014 : Y. Chevrel, A. Cointre, Y.-M. Tran-Gervat (dir.), Histoire des Traductions en Langue Française. XVII E et XVIII ${ }^{E}$ siècles, Lagrasse : Verdier.

Descombes 1987 : V. Descombes, Proust. Philosophie du roman, Paris : Éditions de Minuit, « Critique ».

Dupuy 1793 : Ch.-Fr. Dupuy, «Éloge de Monsieur l'abbé Batteux », Histoire de l'académie des inscriptions et belles-lettres, t. 45, Paris : Imprimerie nationale exécutive du Louvre, 91-106.

Garncarzyk 2017 : D. Garncarzyk, « Originaux, traductions et langue neutre : pistes pour une théorie de la traduction-relais au $18^{\mathrm{e}}$ siècle », TRANS-, $\mathrm{n}^{\circ} 22:\langle\mathrm{http}: / /$ trans. revues.org/1693).

Genette 2004 : G. Genette, Fiction et diction, Paris : Seuil, « Points essais ».

Goujet 1758 : Cl.-P. Goujet, Mémoire historique et littéraire sur le Collège royal de France, Paris : Augustin-Martin Lottin l'aîné, t. II.

Kremer 2008 : N. Kremer, Préliminaires à la théorie esthétique du XVIII siècle, Paris : Kimé.

La Motte 1715 : A. Houdar de La Motte, Réflexions sur la critique, Paris : Du Puis.

Laugier 2015 : S. Laugier, «La vulnérabilité des formes de vie », Raisons politiques, $\mathrm{n}^{\circ}$ 57, Paris : Presses de Sciences Po, 65-80. 〈https://www.cairn.info/revue-raisonspolitiques-2015-1-page-65.htm>, 13/05/2019.

Norman 2011 : L. F. Norman, The Shock of the Ancient, Chicago-London : University of Chicago Press.

Nussbaum 1990 : M. Nussbaum, Love's Knowledge. Essays on philosophy and Literature, Oxford : OUP.

Pouivet 2006 : R. Pouivet, Le Réalisme esthétique, Paris : Presses universitaires de France, "L'interrogation philosophique ».

Rabau 2012 : S. Rabau, Quinze (brèves) rencontre avec Homère, Paris : Belin, « L'Antiquité au présent ».

Tiercelin 2012 : Cl. Tiercelin, « Bouveresse dans le rationalisme français », Agone, $\mathrm{n}^{\circ} 48$, Marseille : Agone, 11-34. 〈https://agone.org/revueagone/agone48/enligne/1/index.html\#debut-chapitre>, 02/06/2017.

Wittgenstein 2004 : L. Wittgenstein, Recherches philosophiques, Paris : Gallimard, «TEL». 
Dimitri Garnkažik

\section{Ima li lirska poezija epistemičku vrednost? Skica unakrsnog čitanja Šarla Batea i Žaka Buvresa}

Rad pruža prvi pokušaj unakrsnog tumačenja pojedinih stavova teoretičara književnosti Šarla Batea (1713-1780) i francuskog analitičkog filozofa Žaka Buvresa (*1940) prema lirskoj poeziji kako bi došao do odgovora na sledeće pitanje iz polja filozofije književnosti: ima li lirska poezija epistemičku vrednost? U uvodu pružamo objašnjenje izbora teme, to jest čitanja i poređenja gledišta dvojice autora, koje se na prvi pogled može učiniti necelishodnim, i predstavljamo metodološki pristup racionalne rekonstrukcije, preuzet iz analitičke filozofije. U prvom delu pažnju posvećujemo (relativnom) mestu koje lirska poezija zauzima u Buvresovom ogledu Znanje književnika (2008), uočavajući smernice koje ovo delo pruža u vezi sa epistemičkim potencijalnom lirske poezije. U drugom delu rada predočavamo kako su u izvesnim aspektima Bateovog Tečaja književnosti (1753) obrađena ova pitanja. U zaključku rada podvučene su prednosti i teorijski potencijali ovakvog postupka unakrsnog čitanja i nagoveštene su smernice za dalju razradu postavljene problematike.

Ključne reči: lirska poezija, racionalizam, Buvres, Bate, kognitivna estetika. 